\title{
Second Harmonic Generation in Generalized Ferroelectric Superlattices
}

\section{Zafer Ozer, Selami Palaz, Amirullah M. Mamedov \& Ekmel Ozbay}

To cite this article: Zafer Ozer, Selami Palaz, Amirullah M. Mamedov \& Ekmel Ozbay (2021)

Second Harmonic Generation in Generalized Ferroelectric Superlattices, Integrated Ferroelectrics, 220:1, 30-38, DOI: 10.1080/10584587.2021.1921532

To link to this article: https://doi.org/10.1080/10584587.2021.1921532

Published online: 01 Dec 2021

Submit your article to this journal 7

山ll Article views: 42

Q View related articles $ᄃ$

View Crossmark data $\nearrow$ 


\title{
Second Harmonic Generation in Generalized Ferroelectric Superlattices
}

\author{
Zafer Ozer ${ }^{a}$, Selami Palaz ${ }^{b}$, Amirullah M. Mamedov ${ }^{c, d}$, and Ekmel Ozbay ${ }^{c}$ \\ ${ }^{a}$ Mersin Vocational High School, Mersin University, Mersin, Turkey; ${ }^{b}$ Faculty of Science, Department of \\ Physics, Haran University, Şanlıurfa, Turkey; ${ }^{C}$ Nanotechnology Research Center, Bilkent University,

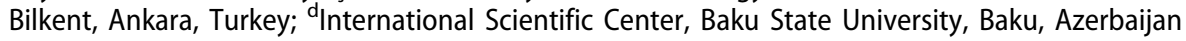

\begin{abstract}
The goal of the work presented in this investigation is to achieve the efficient parametric interaction of narrow beams (in particular second harmonic generation) using a nonlinear (ferroelectric-LiTaO ${ }_{3}$ ) photonic crystal tuned to self-collimation (nondiffractive) regimes. A numerical study of second harmonic generation (SHG) in one-dimensional nonlinear photonic crystals based on a full nonlinear system of equations, implemented by a combination of the method of finite elements and fixed-point iterations, is reported. This model is derived from a nonlinear system of Maxwell's equations that partly overcomes the known shortcoming of some existing models that rely on the undepleted pump approximation. We derive a general solution of SHG in one-dimensional nonlinear photonic crystals structures. Numerical simulations also show that the conversion efficiency of SHG can be significantly enhanced when the frequencies of the fundamental wave are located at the photonic band edges or are assigned to the designed defect states.
\end{abstract}

\section{ARTICLE HISTORY}

Received 23 November 2020

Accepted 25 March 2021

\section{KEYWORDS}

Second harmonic generation; photonic crystal; ferroelectric; finite element method

\section{Introduction}

Nonlinear optical properties in periodic and quasiperiodic structures have been studied for a long time [1-3]. In a number of applications, nonlinear photonic crystal (PC) devices offer a unique, fundamental way of enhancing a variety of nonlinear optical processes, and it may enable $100 \%$ conversion in principle [4]. Efficient numerical methods for second harmonic generation (SHG) in photonic crystals are needed to analyze, design, and optimize these structures. Over the last decade, nonlinear optical (NLO) processes, such as SHG in nonlinear PC, have attracted extensive interest [4-9]. Nonlinear PCs offer fundamental and unique methods of enhancing various NLO processes.

In the present paper, we theoretically investigate the NLO processes in $1 \mathrm{D}$ nonlinear PCs based on aperiodic and quasi-periodic structures, e.g., Fibonacci or Thue-Morse sequences [6-9].

\section{Structure and Calculation Method}

The schematic of the fractal structure used in the present paper is shown in Fig. 1. The structure is aperiodic. We consider a nonlinear material with $N$ layers. The structure is 

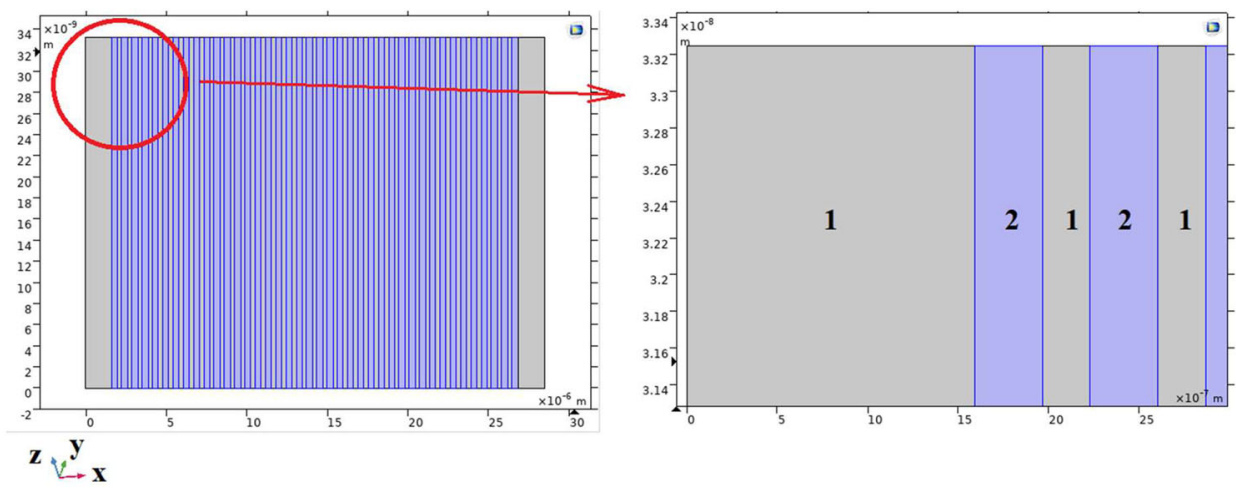

Figure 1. $1 \mathrm{D}$ nonlinear PC structure.

considered along the $x$-direction, for $0 \leq x \leq d$, and the media is considered to be nonmagnetic. Considering that the fundamental frequency and the second harmonic wave (SHW) are given in the TE (transverse electric) polarization, the equations for the SHG will be:

$$
\begin{aligned}
& \frac{d^{2} E_{1}}{d z^{2}}+\left(k_{0} n_{1}\right)^{2} E_{1}=-k_{0}^{2} \chi_{1}^{(2)} \bar{E}_{1} E_{2} \\
& \frac{d^{2} E_{2}}{d z^{2}}+\left(2 k_{0} n_{2}\right)^{2} E_{2}=-2 k_{0}^{2} \chi_{2}^{(2)} E_{1}
\end{aligned}
$$

Where $E_{1}$ and $E_{2}$ are the electric fields for the fundamental frequency (FF) and for the $\mathrm{SH}$ frequency $2 \mathrm{z} . \bar{E}_{1}$ is the complex conjugate, $k_{o}=\omega / c$ is the wave number in vacuum, $c$ is the speed of light in vacuum, $n_{1}$ and $n_{2}$ are the refractive indices of $\omega$ and $2 \omega$, and $\chi_{1}$ and $\chi_{2}$ are two second order elements of the nonlinear susceptibility tensors for $\omega$ and $2 \omega$.

The purpose of the SHG is to start with a laser wherein the emitting wavelength is $1.064 \mu \mathrm{m}$ then double the wavelength to green at $0.532 \mu \mathrm{m}$. With the same producing method, it is also possible to generate ultraviolet (UV) light at $0.355 \mu \mathrm{m}$.

We assume that the nonlinear PC composed of periodically stocked nonlinear material $N$-dielectric $L$ layers that is periodic in the $x$ direction where $0 \leq x \leq d$.

In this study, we showed how to generate a second harmonic frequency by injecting fundamental frequency light into the nonlinear photonic crystal with coupled two frequency-domain interfaces that are a first interface with $f=c / \lambda_{o}$ frequency, and a second interface with the second harmonic frequency $2 f$ and wavelength $\lambda_{1}=c / 2 f$.

In Fig. 1, a nonlinear photonic crystal (PC) consists of air (1) and nonlinear material (2) in which refractive indices are $n L=1$ and $n H=1.42857$, respectively. The width of the strips is $d L=\lambda_{o} /(4 n L)$ and $d H=\lambda_{o} /(2 n H), 1 \mathrm{D}$ unit cell thickness $a=d L+d H$ where the reference wavelength is $\lambda_{o}=1.064 \mu \mathrm{m}$ as in [6]. The width of $1 \mathrm{D} \mathrm{PC}$ is $\lambda_{1} / 16$ and the length is approx. $40 \times \lambda_{o}$.

We assumed that the TE mode electromagnetic wave propagates in the $x$ direction. We set the scattering boundary condition left boundary of the PC as the incident electric field is $E_{z}=\sqrt{\frac{2 I}{\varepsilon_{0} c}}$ where $I=30 \mathrm{MW} / \mathrm{m}^{2}$ are the incident fundamental intensity, $c$ speed of light, and $\varepsilon_{0}$ the permittivity of vacuum. 
Table 1. Fibonacci sequence.

\begin{tabular}{llcc}
\hline Fibonacci sequence & $S_{j+1}=S_{j-1}, S_{j}$ & Fibonacci number & Generation number \\
\hline$S_{0}$ & $\mathrm{~N}$ & 1 & 0 \\
$S_{1}$ & $\mathrm{~L}$ & 1 & 1 \\
$S_{2}$ & $\mathrm{NL}$ & 2 & 2 \\
$S_{3}$ & $\mathrm{LNL}$ & 3 & 3 \\
$S_{4}$ & $\mathrm{NLLNL}$ & 5 & 4 \\
\hline
\end{tabular}

For the fundamental interface, we applied

$$
P_{1 z}=2 \chi E_{2 z} E_{1 z}^{*}(\text { polarization })
$$

For the second harmonic frequency interface, we applied

$$
P_{2 z}=\chi E_{1 z}^{2} \text { (polarization) }
$$

The nonlinear coefficient value for the typical nonlinear material is on the order of $\chi=1 \times 10^{-24}-1 \times 10^{-21} \mathrm{C} / \mathrm{V}^{2}$ but for simplification, we took the nonlinear coefficient as $\chi=1 \times 10^{-18} \mathrm{C} / \mathrm{V}^{2}$ as shown in Eqs. (3) and (4).

\section{Fibonacci Nonlinear Photonic Crystal}

To compare the SHG performance with nonlinear Photonic Crystal (PC), we designed a 5th order quasi-periodic multilayer nonlinear Fibonacci Photonic Crystal [3] with the same parameters as in nonlinear (NL) PC.

Fibonacci sequence descripted by the $S_{j+1}=S_{j^{-} 1}+S_{j} j \geq 1$ formula where $S_{j}$ is a $j$.th order Fibonacci sequence. If you assume $S_{0}=\mathrm{N}$ and $S_{1}=\mathrm{L}$, and the Fibonacci sequence after $j$ iteration, the first 4 generations are as shown in Table 1.

\section{Numerical Results and Discussion}

Numerically, we tested our method on a simple structure made of nonlinear material with $N$ layers (Fig. 1). We show the linear transmission spectrum versus the free space wavelength for an $\mathrm{N}$-layer conventional PC (at the fundamental and $\mathrm{SH}$ frequencies) in Fig. 2. We calculated the FF and $\mathrm{SH}$ fields at a number of frequencies around maximum transmission peak situated close to the low-frequency PBE (Photonic Band edges): $\Omega=$ $0.57\left(\omega / \oplus_{o}\right)$. The second order NL coefficient of $\mathrm{LiTaO}_{3}$ layers is assumed to be $\chi^{(2)}$ $=89.5 \times 10^{-18} \mathrm{C} / \mathrm{V}^{2}$ and the refractive index of $\mathrm{LiTaO}_{3}$ at the second harmonic frequency is 2.26 . The incident wave is the mode with $E=10^{6} \mathrm{~V} / \mathrm{m}$. If the perfect structure is affected in any way, the result of any permitted states at PBG may produce a rise of the field in the defect.

The absolute values of the generated SH wave at $x=d$ is shown versus the free space wavelength at the fundamental frequency in Fig 3. At $\lambda=1.13 \mu \mathrm{m}$, the maximum obtained is $354 \mathrm{~V} / \mathrm{m}$.

As is well known, if the conventional structure is frustrated in the appropriate manner, some of the resulting allowed states within the PBG can give rise to the field enhancement in the structural defect (or periodicity). We introduce a defect by changing the periodicity of the structure. 


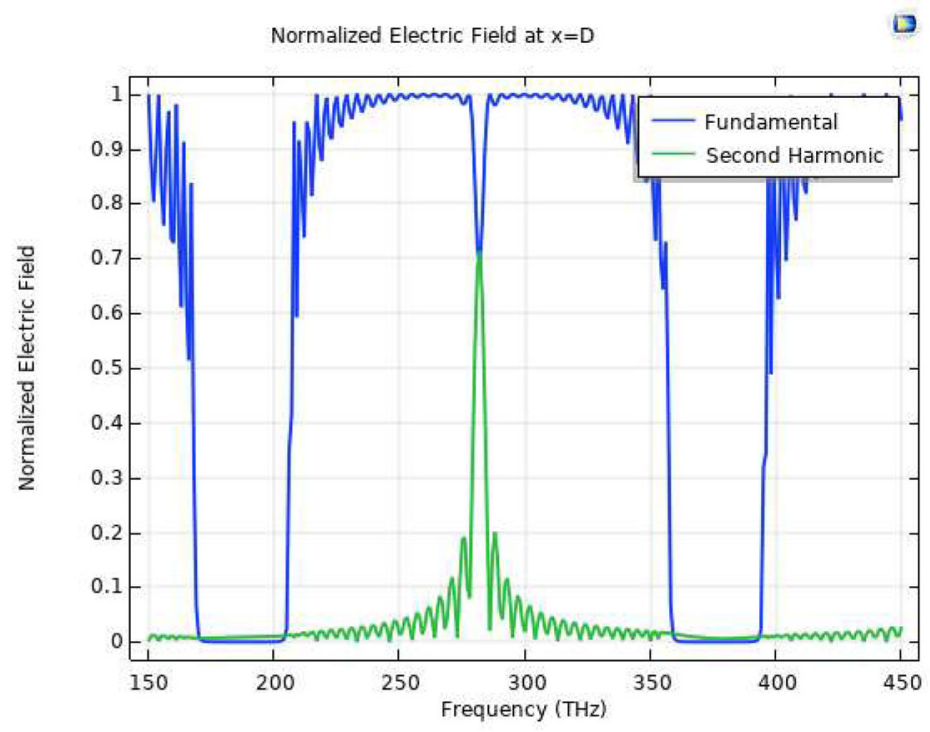

Figure 2. Normalized electric field at $x=d$ in nonlinear PC.

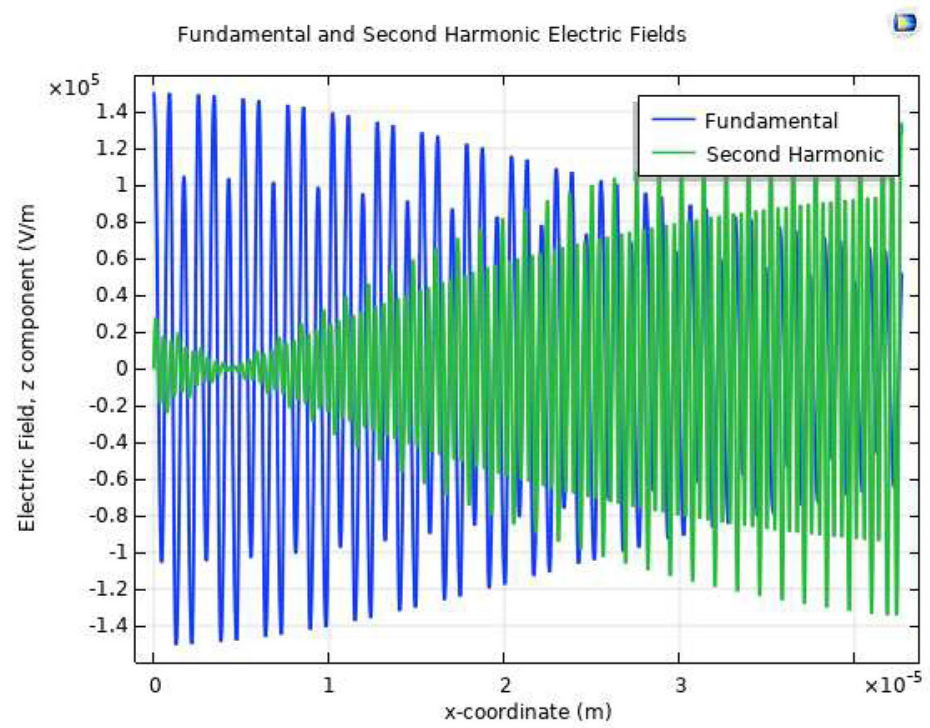

Figure 3. Fundamental and second harmonic electric fields in nonlinear conventional PC.

The SHG conversion efficiency in nonlinear PCs can be larger when we simultaneously adjust the frequencies of both the FW and SHW to the frequencies located at the PBEs. In Fig. 3, we represented the absolute values of the FF and SH fields. The FF is decreased with more than one order of magnitude compared to the peak value outside of the structure. Therefore, the conversion efficiency of SHG should significantly increase. Because of the wavelength of the SH signal, which is half of the pumped wave, there are two maximum intensities of the $\mathrm{SH}$ wave within each high index layer. 


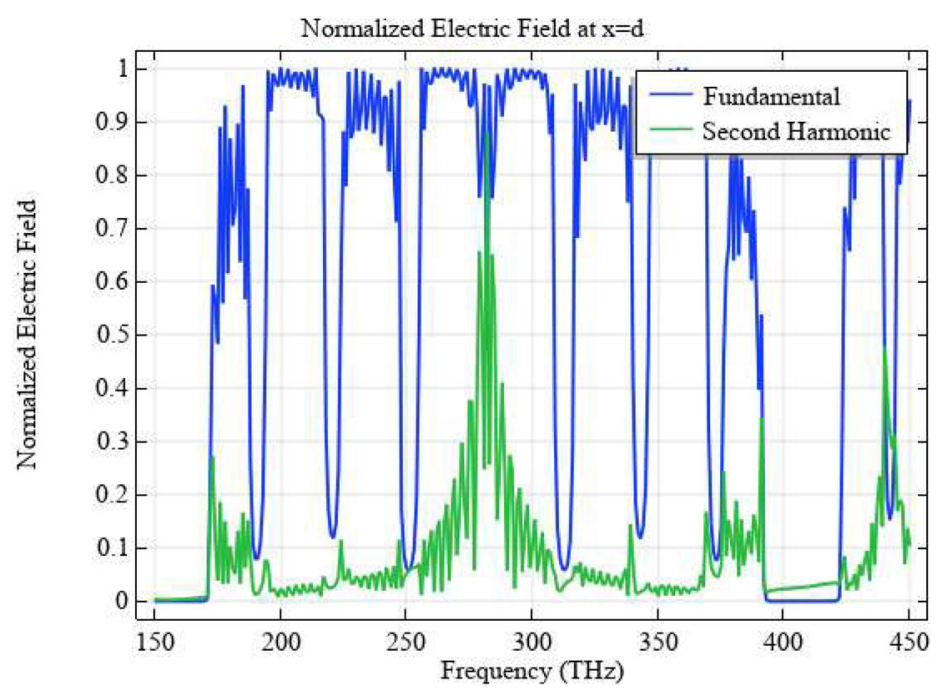

Figure 4. Normalized electric field at $x=d$ in Fibonacci nonlinear PC.

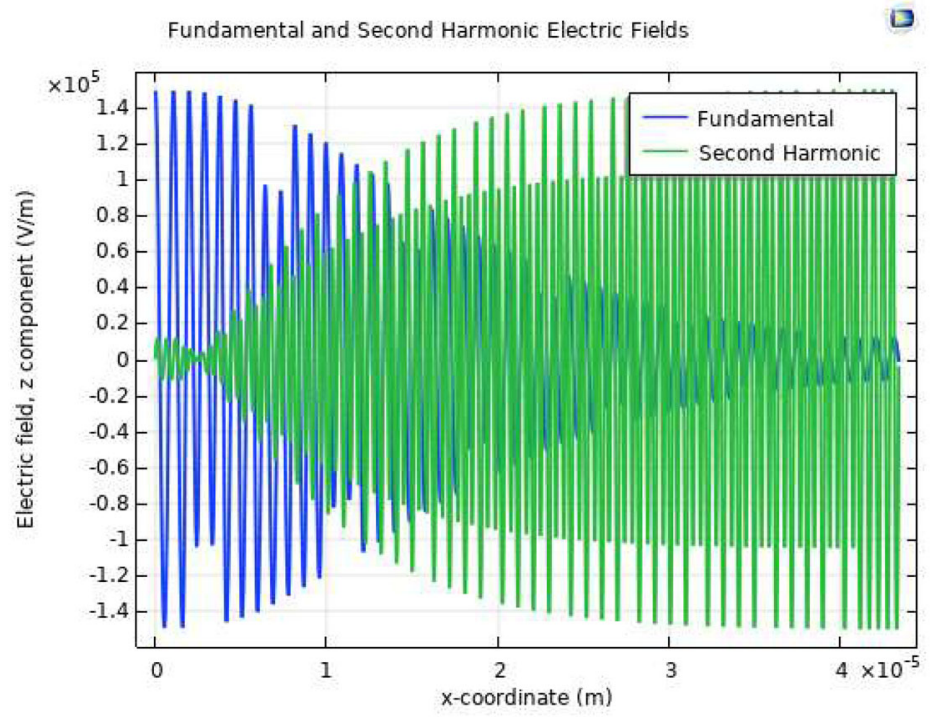

Figure 5. Fundamental and second harmonic electric fields in Fibonacci nonlinear PC.

We have also designed a new PC with a structural defect (Fibonacci PC in the form of $S_{i}$, where $S$ represents different Fibonacci sequences) and calculated the transmittance of fundamental and SHW (Figs. 4 and 5). We chose $\mathrm{LiTaO}_{3}$ and air with nonlinear and linear layers, respectively. All of the structural parameters of the $1 \mathrm{D}$ Fibonacci PC structure are the same as for the conventional PC structure.

The second order nonlinear coefficient of the $\mathrm{LiTaO}_{3}$ layers is assumed to be $\chi=128 \times 10^{-18} \mathrm{C} / \mathrm{V}^{2}$ and the refractive index of $\mathrm{LiTaO}_{3}$ for the $\mathrm{SH}$ frequency is 2.28. The incident wave is $E_{i}=10^{6} \mathrm{~V} / \mathrm{m}$. Because of the nonlinearity of the material, SHG can grow significantly. We introduced a defect by changing the structure of PC from 
conventional to fractal. We studied the generation of the FFW and SHW around the fundamental wavelength.

The absolute value of the $\mathrm{FF}$ and $\mathrm{SH}$ fields at the fundamental wavelength in this defect structure is shown in Figs. 4 and 5. The fundamental frequency field and the SH field from the fractal structure grow simultaneously.

\section{Time Domain Analysis}

With the same refractive index and same strip thickness as in the frequency domain analysis, we conducted time-domain analysis with 35 periods of stacks of nonlinear PC $\left(21 \times \lambda_{0}\right.$ length) and 6 . order Fibonacci PC. Nonlinear PC ( $\cong 16 \times \lambda_{0}$ length).

In our model, the beam in transverse Gauss form in the $y$ direction and travels in the $x$ direction through the electric field polarized in the $z$ direction. In a $2 \mathrm{D}$ medium, for a Gaussian beam whose radius is not too small relative to its wavelength $\left(\omega_{0}>\lambda_{0}\right)$, the solution of time harmonic Maxwell equations can be approximately obtained by the solution of the paraxial wave equation given in Eqs. (1)-(2).

$$
E(x, y, z)=E_{0} \sqrt{\frac{\omega_{0}}{\omega(x)}} e^{-\left(\frac{y}{\omega(x)}\right)^{2}} \cos \left(\omega t-k x+\eta(x)-\frac{k y^{2}}{2 R(x)}\right) e_{z}
$$

Where

$$
\begin{gathered}
\omega(x)=\omega_{0} \sqrt{1+\left(\frac{x}{x_{0}}\right)^{2}} \\
\eta(x)=\frac{1}{2} \arctan \left(\frac{x}{x_{0}}\right) \\
R(x)=x\left(1+\left(\frac{x_{0}}{x}\right)^{2}\right)
\end{gathered}
$$

In these equations, $k$ is the wave number, $y$ is the in-plane transverse coordinate, $\omega$ is the angular frequency, and $\omega_{0}$ is the length of the narrowest part of the gaussian beam. The laser beam could model as a time pulse that produces a wave packet with the Gaussian frequency spectrum [8].

The polarization matrix $P$ in Eq. (6) describes the nonlinear properties of the material for SHG. We only used the $\chi_{33}$ parameter as $10^{-17} \mathrm{C} / \mathrm{V}^{2}$ to avoid the long computation time and large distance of the model where most of the material is in the $10^{-22} \mathrm{C} / \mathrm{V}^{2}$ range.

$$
P=\left[\begin{array}{llllll}
\chi_{11} & \chi_{12} & \chi_{13} & \chi_{14} & \chi_{15} & \chi_{16} \\
\chi_{21} & \chi_{22} & \chi_{23} & \chi_{24} & \chi_{25} & \chi_{26} \\
\chi_{31} & \chi_{32} & \chi_{33} & \chi_{34} & \chi_{35} & \chi_{36}
\end{array}\right]\left[\begin{array}{c}
E_{x}^{2} \\
E_{y}^{2} \\
E_{z}^{2} \\
2 E_{z} E_{y} \\
2 E_{z} E_{x} \\
2 E_{x} E_{y}
\end{array}\right]
$$

When the mesh size defines to resolve, it is also considered a second harmonic wavelength $\left(\lambda_{1}\right)$ besides the fundamental wavelength $\left(\lambda_{0}\right)$. The maximum element size is 


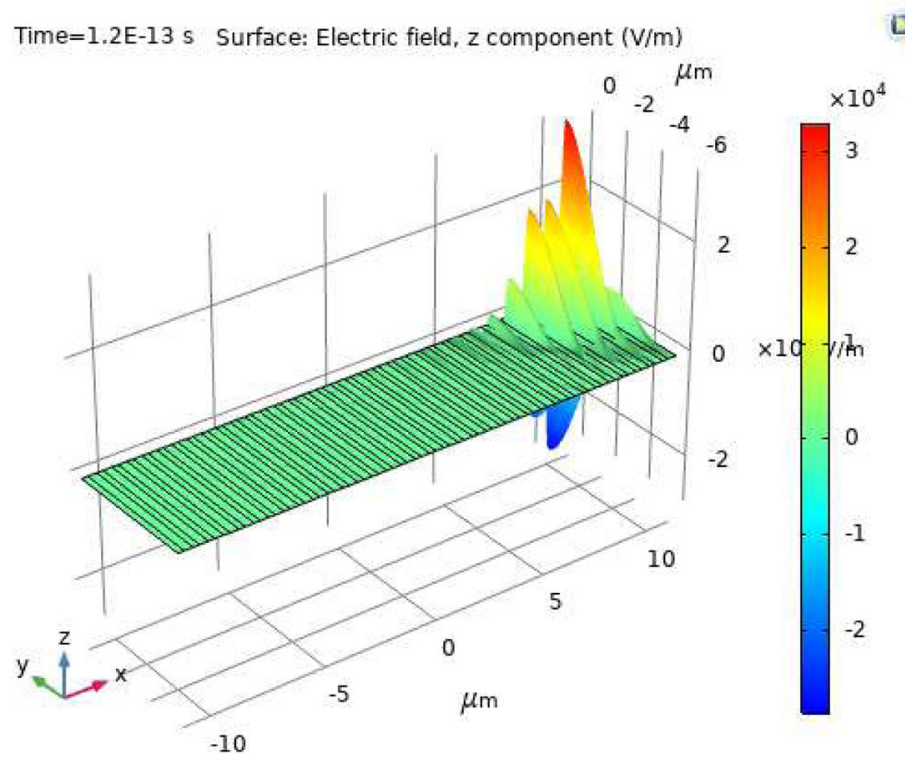

Figure 6. After $120 \mathrm{fs}$, the pulse wave has reached the output boundary.

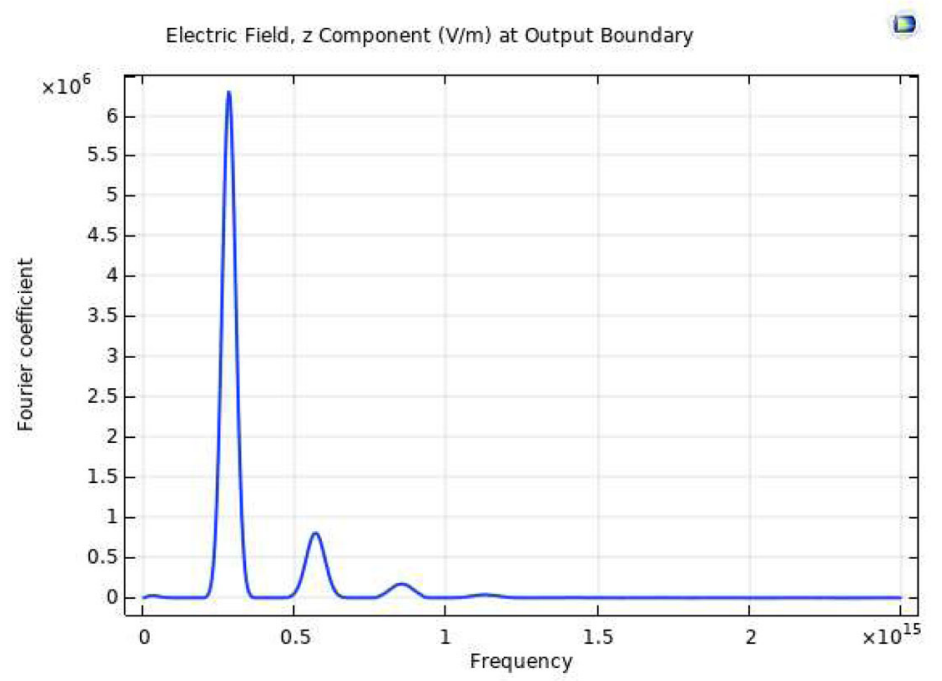

Figure 7. The Fourier transform of the time pulsing wave at the output boundary in nonlinear PC.

defined as $h_{\text {max }}=\lambda_{1} / 6$ and the time step as tstep $=C F L^{*} h_{\text {max }} / c$ where $C F L$ is the Courant, Friedrichs, and Lewy number that is generally between $0.1-$ and 0.2 and $c$ is the vacuum speed of light.

The SHG can be calculated by a pulse wave travel along the nonlinear PC. In the time domain calculation, the pulse wave should enter, pass, and disappear from the PC. As seen in Fig. 6, a pulse wave has reached the output boundary and disappeared after 120 fs later. 


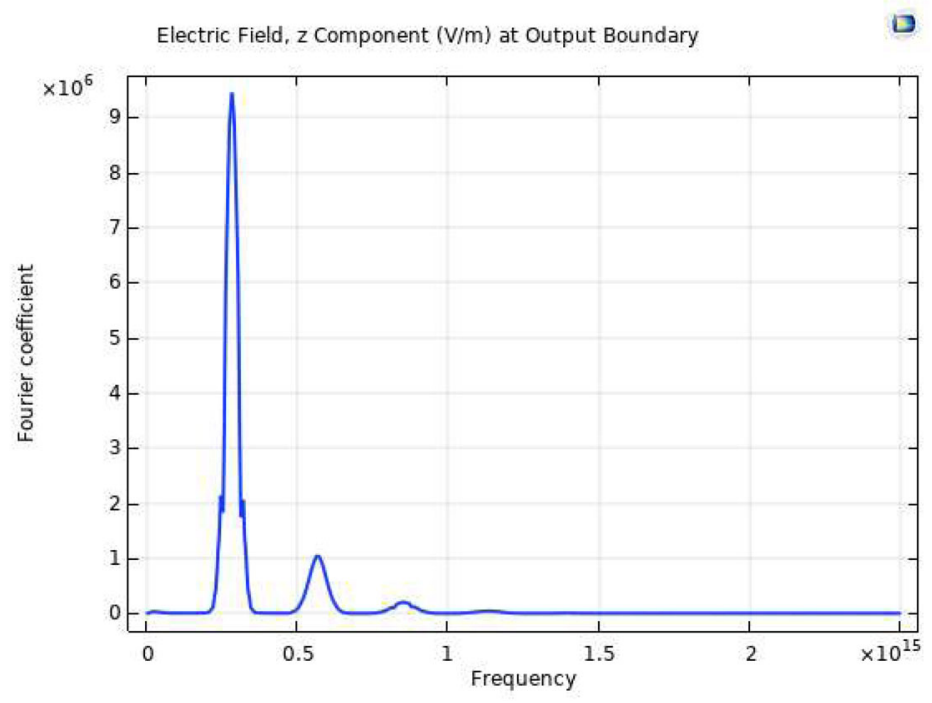

Figure 8. The Fourier transform of the time pulsing wave at the output boundary in 6th order Fibonacci PC.

The second harmonic component of the electric field at the output boundary can be obtained using Fourier analysis. In Figs. 7 and 8, the small peak on the right side of the large peak shows the second harmonic generation in nonlinear PC and 6th order Fibonacci PC, respectively.

It is clearly seen in Figs. 7 and 8 that, although Fibonacci PC has the shortest length $\left(\cong 16 \times \lambda_{0}\right)$ compared to nonlinear PC $\left(21 \times \lambda_{0}\right)$, its SHG performance is better than nonlinear PC.

\section{Conclusion}

In summary, we have performed a numerical study on the SHG in a novel $1 \mathrm{D}$ Fibonacci $\mathrm{LiTaO}_{3}$ superlattice. The full nonlinear SHG problem is solved by a method of finite elements coupled with a fixed-point iteration. We obtained two maximum intensities of the SHW within each high index layer that were in contrast to the FW peak. By using time domain and Fourier analysis, we calculated the second harmonic component of the electric field as a pulse wave traveling along the nonlinear PC. Our calculations show that the pulse wave has reached the output and disappeared after more than $100 \mathrm{fs}$ later.

\section{References}

1. S. V. Gaponenko, Introduction to Nanophotonics (Cambridge University Press, New York, 2010).

2. E. Shamonova, World Scientific Handbook of Metamaterials and Plasmonics (World Scientific, University of Oxford, Singapore, 2018).

3. A. Rostami and S. Matloub, Exactly solvable inhomogeneous Fibonacci-class quasi-periodic structures (optical filtering), Opt. Comm. 247 (4-6), 247 (2005). DOI: 10.1016/j.optcom. 2004.11.105. 
4. D. G. Popesku et al., Second harmonic generation in photonic crystals: Numerical simulation, JOAM 14 (3/4), 356 (2012).

5. J. Yuan, Computing for second harmonic generation in $1 \mathrm{D}$ nonlinear photonic crystals, Opt. Comm. 282 (13), 2628 (2009). DOI: 10.1016/j.optcom.2009.03.049.

6. X. Cai and X. Xuan, Optical harmonic generation in Fibonacci dielectric superlattice of $\mathrm{LiNbO}_{3}$, Opt. Comm. 240 (1-3), 227 (2004). DOI: 10.1016/j.optcom.2004.06.018.

7. R. E. Slusher and B. J. Eggleton, Nonlinear Photonic Crystal (Springer Verlag, Berlin, 2003).

8. M. Scalora et al., Pulsed second-harmonic generation in nonlinear, one-dimensional, periodic structures, Phys. Rev. A. 56 (4), 3166 (1997). DOI: 10.1103/PhysRevA.56.3166.

9. S. Simsek et al., SbSI based photonic crystal superlattices: band structure and optics, IOP Conf. Ser.: Mater. Sci. Eng. 77, 012020 (2015). DOI: 10.1088/1757-899X/77/1/012020. 